Article

\title{
Subwavelength Hexahedral Plasmonic Scatterers: History, Symmetries, and Resonant Characteristics ${ }^{\dagger}$
}

\author{
Dimitrios Tzarouchis *D, Pasi Ylä-Oijala and Ari Sihvola \\ Department of Electronics and Nanoengineering, Aalto University, Maarintie 8, P.O. 15500, Espoo, Finland; \\ pasi.yla-oijala@aalto.fi (P.Y.-O.); ari.sihvola@aalto.fi (A.S.) \\ * Correspondence: dimitrios.tzarouchis@aalto.fi; Tel.: +358-503652771 \\ $+\quad$ This paper is an extended version of our paper published in Tzarouchis, D.C.; Ylä-Oijala, P.; Sihvola, \\ A.Plasmonic eigenmodes on regular nano-polyhedra: Case study of electrostatic and dynamic resonant \\ effects on rounded superquadric solids. 2018 12th Int. Congr. Artif. Mater. Nov. Wave Phenom., 2018, \\ pp. 401-403.
}

Received: 31 January 2019; Accepted: 20 February 2019; Published: 25 February 2019

\begin{abstract}
In this work, we investigate the resonant characteristics of hexahedral (cubical) inclusions at the plasmonic domain. After an introduction to the notion of superquadric surfaces, i.e., surfaces that model various versions of a rounded cube, we present the main resonant spectrum and the surface distributions for two particular cases of a smooth and a sharp cube in the plasmonic domain. We present a historical comparative overview of the main contributions available since the 1970s. A new categorization scheme of the resonances of a cube is introduced, based on symmetry considerations. The obtained results are compared against several recent works, exposing that the higher-order modes are extremely susceptible to both the choice of sharpness of the cube and the modeling mesh. This work can be readily used as a reference for both historical and contemporary studies of the plasmonic aspects of a cube.
\end{abstract}

Keywords: localized plasmonic resonances; plasmonic particles; plasmonic cube; superquadric surfaces; electrostatic scattering; history of plasmonic cubes

\section{Introduction}

Many radiation harnessing applications, in principle, acquire their scattering properties through their building-block constituents. This can be seen as a demonstration of the emergence principle in electromagnetics [1]. The scattering properties of a single scatterer are crucial to understanding and reinventing all the emergent phenomena and in the design of novel composites with exotic and on-demand functionalities. Subsequently, the material and morphological setup plays an essential role in this direction.

Apart from the plethora of light control applications that use these emerging collective effects [2], also individual resonating particles exhibit a great variety of interesting physical properties and mechanisms, e.g., Fano resonances and enhanced Purcell factors in single particles and nanoantennas [3,4]. Given a certain plasmonic material, such as gold or silver, at the optical-IR range, the study of the enabled physical mechanisms reduces to the study of the morphological effects over the scattering mechanisms within the classical electrodynamic domain. For instance, a sphere and an infinite circular cylinder are canonical examples of particles for which there exists great amount of electromagnetic scattering analysis [5,6]. These are two of the very few examples that allow a closed-form analytical solution through either electrostatic or electrodynamic scattering approach; an ocean of analytically unsolvable particles remains unexplored.

One characteristic example of a particle with very interesting properties without, for the moment, a closed-form solution is the cube. Cubes can grow either naturally or through sophisticated artificial 
processes, and cubes have been extensively used as examples of plasmonic particles with enhanced field features in the vicinity of their edges and vertices [4,7-9]. The first numerical attempts treating the cube (hexahedron) case, and its plasmonic resonances, were given by Gelder et al. [10] and Fuchs [11] in the early 1970s. Since that time, the cube and its scattering characteristics have remained a fruitful research topic, with steadily increasing interest over the recent years [12-27].

This work is an extension of our results presented in the Metamaterials 2018 conference [28]. There, the main attention of the discussion was towards both electrostatic and eigenmode characteristics of regular polyhedra, the Platonic solids, including the hexahedron, i.e., the cube. During and after this conference presentation, we received several comments and suggestions for further studies, which revealed the interest of the metamaterials community for the scattering properties of a cube. In particular, questions were raised on how realistic can the study of a cube be, the relation between the sharpness of a cube and the strength and position of the main plasmonic resonance, and whether there are any available analytical perspectives regarding the cube.

These valuable inputs revealed clearly that despite a long history and the number of recent works, a systematic comparative study of the plasmonic features of a cube is still missing. Therefore, the present report aims at narrowing this gap by introducing a detailed analysis for the plasmonic resonant characteristics of a cube from an electrostatic perspective, substantiated by a historical comparative analysis between the most relevant works found to date. The analysis includes five "historical" articles (between 1972 and 2004) [10-12,29,30], and four more recent studies (after 2011) [13-16], all compared with the results presented here. Of course, this reference list is not exhaustive, but rather focuses within certain applied physics, radio science, material, and chemistry communities, where the study of plasmonic resonances on single subwavelegth particles is frequently considered.

The article is structured as follows: First, in Section 2 a brief theoretical discussion about the superquadric surfaces is presented. This is a necessary step towards the proper modeling of a rounded cube. After that, Section 3 contains the main results for two sharpness setups for the cube, i.e., a "smooth" and a "sharp". Both are numerically modeled with a regular and a refined mesh. The computations are based on a standard, surface integral equation scheme for the electrostatic potential presented in [12]. Section 4 compares the results against the most seminal references in the literature. The historical articles cover almost four decades of research on the subject. Since these references lack robust visual surface potential representation, the comparison and assessment is only based on the relative position of the resonances.

After the historical section, a new categorization scheme is introduced in Section 5 for an efficient classification of the observed resonances. This categorization scheme is based on symmetry considerations. Finally, Section 6 discusses the extreme sensitivity of the higher-order modes to the choice of both the rounding and meshing setups. Moreover, this section includes a thorough comparison between more recent results (2011-today, [13-16]) that contain calculations of both the resonant spectra and the surface potential distributions for the most pronounced resonances. Surprisingly, the comparison reveals a disagreement of the surface potential distribution for certain higher-order modes. We conclude this section by carefully considering the effects of the mesh density and roundness to the near field features, hence resolving these reported disagreements.

\section{Superquadric Surfaces and the Cube}

The systematic analysis of arbitrarily shaped scatterers with a potential-based (electrostatic) integral-equation methodology requires the proper discretization of the scatterer's surface [12]. In modern computer graphics, computer vision, and pattern recognition sciences there is an important family of parametric surfaces, so-called superquadric surfaces.

Originally, two-dimensional superelliptical curves (Lamé curves) where first described by Lamé [31,32]. In the 1960s, Hein introduced the surface equivalent of the Lamé curves for 3D design purposes. He named these surfaces superellipsoids or superspheres [32,33]. In 1981, Barr generalized the notion of superellipses in a more systematic way to the general family of superquadrics [32,34-36]. 
An even more general family that includes the superquadric surfaces are the hyperquadrics, introduced by Hanson in 1988 for computer graphic applications [37].

A superquadric shape can be straightforwardly generated by the following expression

$$
f(x, y, z)=\left|\frac{x}{a}\right|^{2 p}+\left|\frac{y}{b}\right|^{2 p}+\left|\frac{z}{c}\right|^{2 p}=1,
$$

where $x, y$, and $z$ are the Cartesian coordinates, $p$ is the rounding factor, and $a, b, c$ are the axis scaling factors.

Let us focus on the case $a=b=c=d / 2$, where $d$ is the diameter of a sphere. For $p=1$ we obtain a sphere, $p \rightarrow \infty$ defines a cube, and the range between $p \in(1,+\infty)$ gives a form that resembles a "rounded cube", as illustrated in Figure 1. Important geometric properties of these surfaces are the cross sectional area (along the Cartesian axes $\mathbf{u}_{x}, \mathbf{u}_{y}, \mathbf{u}_{z}$ ) and volume. The expression for the area of a superquadric of diameter $d$ is

$$
C_{\mathrm{g}}=\frac{d^{2}}{2 p} \mathcal{B}\left(\frac{1}{2 p}, \frac{1}{2 p}+1\right)
$$

where $\mathcal{B}$ is the beta function. For $p=1$ we have $C_{\mathrm{g}}=\pi d^{2} / 4$, since $\mathcal{B}(0.5,1.5)=\pi / 2$. Similarly, the volume reads

$$
V=\frac{d^{3}}{4 p^{2}} \mathcal{B}\left(\frac{1}{p}, \frac{1}{2 p}+1\right) \mathcal{B}\left(\frac{1}{2 p}, \frac{1}{2 p}\right)
$$

Again, $p=1$ gives the volume of a sphere, $V=\pi d^{3} / 6$, since $\mathcal{B}(1,1.5)=2 / 3$ and $\mathcal{B}(0.5,0.5)=\pi$.

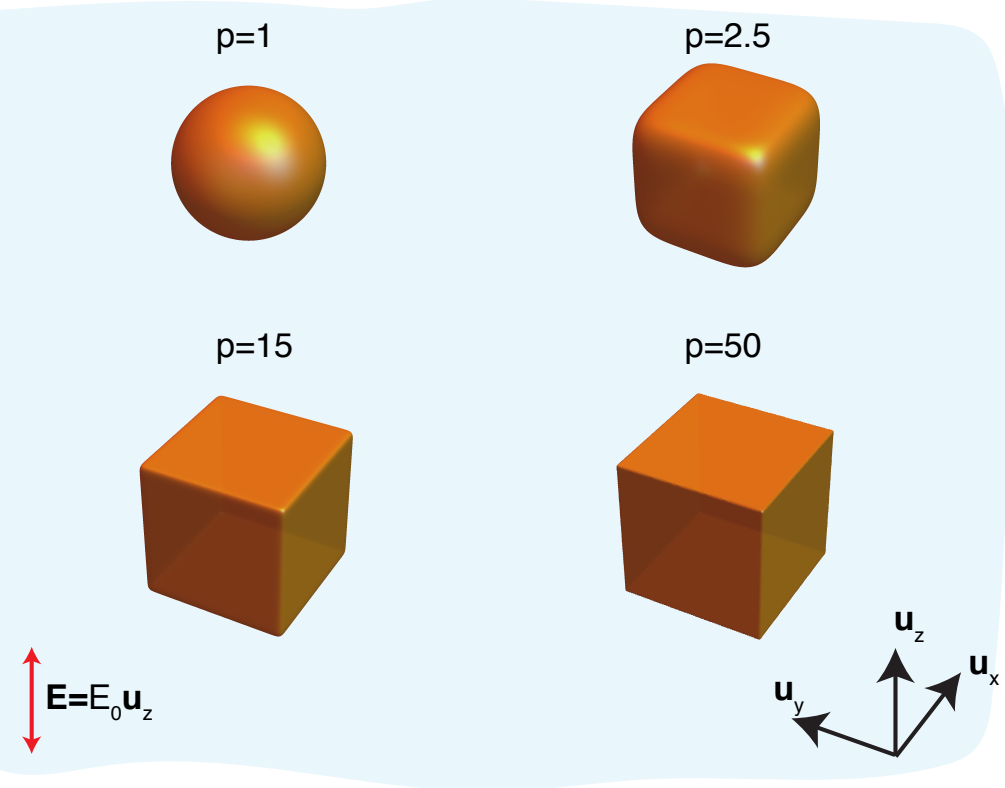

Figure 1. The transformations of a superquadric sphere for increasing values of the rounding factor $p$. The bottom left and bottom right cases depict the studies cases used in this work: a "smooth" $p=15$ and a "sharp" $p=50$ cube. The figure indicates a constant $z$-directed electrostatic excitation $\mathbf{E}=E_{0} \mathbf{u}_{z}$.

A more intuitive picture of the $p$-dependence for both the area and the volume of the superquadrics for the limiting cases can be derived by inspecting the approximative expressions of (2) and (3). Table 1 summarizes the first Taylor expansion terms for the two characteristic cases, i.e., a sphere $(p=1)$ and a cube $(p \rightarrow \infty)$. 
Table 1. Asymptotic behavior of the volume and area of a superquadric close to a sphere $(p=1)$ and a cube $(p \rightarrow \infty)$.

\begin{tabular}{lcc}
\hline Superquadric Cases & $V \approx$ & $C_{\mathrm{g}} \approx$ \\
\hline$p \approx 1$ & $\frac{1}{6} \pi d^{3} p$ & $\frac{\pi d^{2}}{4}\left(1+\ln (4 / e)^{p-1}\right)$ \\
\hline$p \rightarrow \infty$ & $d^{3}\left(1-\frac{\pi^{2}}{8 p^{2}}\right)$ & $d^{2}\left(1-\frac{\pi^{2}}{24 p^{2}}\right)$ \\
\hline
\end{tabular}

\section{Plasmonic Spectra of Smooth $(p=15)$ and Sharp $(p=50)$ Sube}

The aforementioned superquadric surface parametrization enables the study of a whole family of shapes that can be defined continuously for different values of the power factor. In the following we compute the electrostatic (plasmonic) polarizability spectra of two particular cases, i.e., a smooth and a sharp version of a rounded cube with $p=15$ and $p=50$, respectively (Figure 1 ).

In previous works rounded cubes have been simulated: Zhang et al. [13] and Cortie et al. [14] calculated the spectrum of a smooth cube. For example, Cortie analyzed a superquadric cube with $p=10$. On the other hand, Hung et al. [15] and Klimov et al. [16] simulated sharp versions of the cube. Therefore, the choice of both $p=15$ and $p=50$ covers fairly well the aforementioned cases.

For both cases considered in this work we implement two meshing schemes in the discretization of the integral equation, i.e., a regularly distributed mesh and an edge-vertex refined one. These can be seen in the insets in Figures 2 and 3. These meshes contain the same amount of elements distributed in two different ways: (a) the elements of the regular mesh are distributed uniformly while (b) the refined mesh incorporates more elements closer to the edges and vertices of the cube. This choice can help us to uncover any mesh-related features, such as any differences of the surface potential distribution or resonant shifting effects.

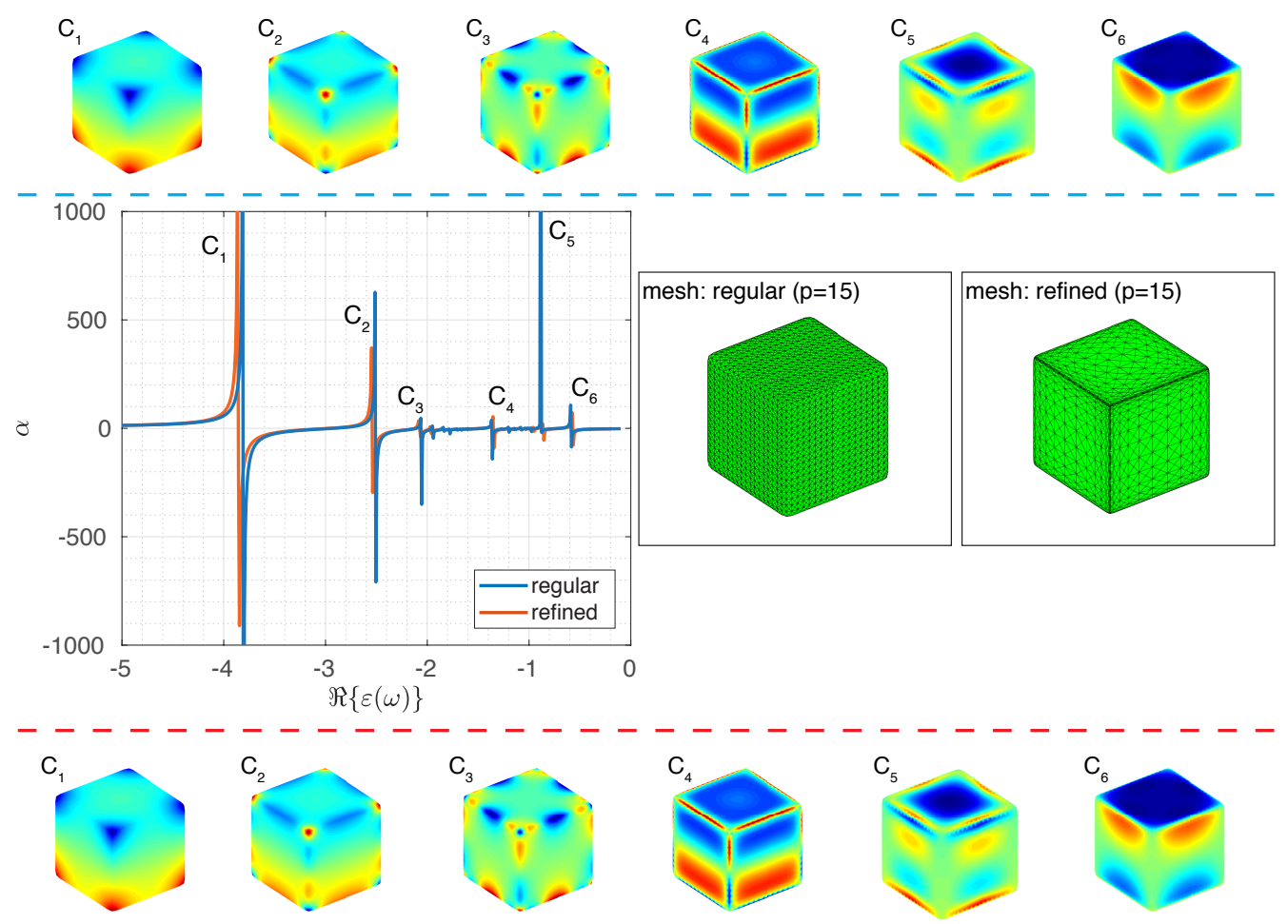

Figure 2. The absolute value of the polarizability at the $\varepsilon \in[-5,0]$ domain of a smooth cube with $p=15$. The color figures at the top color depict the surface potential of the six most pronounced resonances $C_{1}-C_{6}$, for the regular mesh, while at the bottom the same surface potential are shown for the refined mesh. Blue and red colors indicate negative and positive values of the potential. The inset figure illustrates the used meshes, i.e., regular and refined ones. 


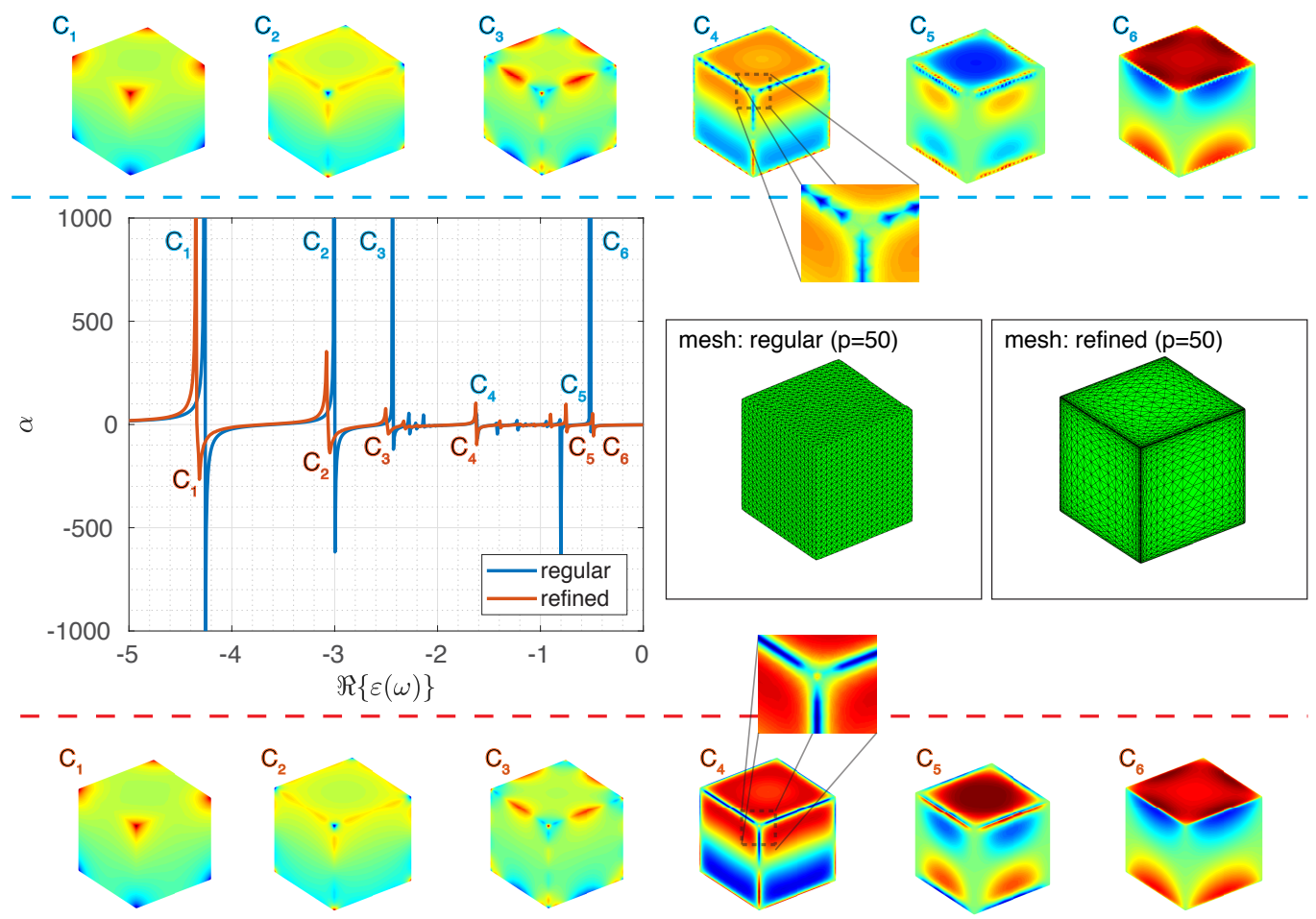

Figure 3. As in Figure 2, the absolute value of the polarizability at the $\varepsilon \in[-5,0]$ domain for the sharp cube $(p=50)$. The color figures at the top depict the surface potential of the six most pronounced resonances $C_{1}-C_{6}$, extracted with a regular mesh, while the bottom figures are extracted with a refined mesh. The $C_{4}$ resonance is actually different between the two mesh setups (inset sketches top and bottom). Note also the disagreement of the $C_{3}$ resonance between the smooth case (Figure 2) and the sharp case (here) [15]. The observed inconsistencies provide evidence that the higher-order modes residing at $\varepsilon \in(-3,-1)$ are sensitive to both the mesh and the sharpness of the cube.

Likewise, Figures 2 and 3 also depict the polarizability of the cube as a function of the permittivity, and the surface potential distribution of each of the four cases. The polarizability spectra contain rich resonant structure. The position of the resonances for all cases are summarized in Table A1 available in the Appendix A.

All results are extracted for an ideal lossless case. Hence the sweep parameter is the relative permittivity of the cube $\varepsilon$, which is a real number. Admittedly, this is a simplification, since in many realistic cases the plasmonic resonances occur in lossy and dispersive materials. Here we denote this fact by representing the permittivity either as $\varepsilon$ or as $\Re\{\varepsilon(\omega)\}$. However, the lossless electrostatic analysis presented here embraces the plasmonic resonances adequately, exposing the main resonant features. This is supported by the fact that the plasmonic resonances occur in deeply subwavelength scatterers. Hence the electrostatic approximation effectively captures these resonant effects.

\subsection{Case $p=15$}

The results for a smooth cube are shown in Figure 2. There, we observe the existence of at least six resonances, namely $C_{1}-C_{6}$, within the $\varepsilon \in[-5,0]$ interval. In addition, one can observe extra resonances between $C_{3}$ and $C_{5}$ peaks. For both meshes the number of used elements was 17 per edge, while the $\varepsilon$ sweep parameter included 600 values, i.e., a step with width of 0.0083 . For this case the regular and refined meshes lead to similar spectra and similar surface potential distributions.

The first observed resonance $\left(C_{1}\right)$ clearly corresponds to a potential distribution of a dipole which is the most dominant mode, verified multiple times in the literature. Since resonances $C_{2}$ and $C_{3}$ reveal a distribution along the edges of the cube, all $C_{1}-C_{3}$ can be categorized within the same edge-type of resonances. The $C_{4}$ resonance exhibits both edge and face variations, i.e., an edge-face 
(or edge-hedra) type, while the resonances $C_{5}$ and $C_{6}$ exhibit no edge variations but rather only face-type variations. These observations lead to a simple categorization based on the exhibited type of variations. The reported resonances are excited by a constant field. Different types of excitations can reveal different types of resonances, see for example the dark mode analysis in [13]. An excitation independent eigenmode analysis can expose the possible solutions for any possible excitation $[38,39]$.

\subsection{Case $p=50$}

The polarizability spectrum, the surface potential distribution, and the two different meshes for the sharper cube $(p=50)$ are depicted in Figure 3. As before, we observe at least six prominent polarization enhancement peaks. Comparison between the smooth and sharp cube exposes two different shifts of the resonances. The observed resonances below $\varepsilon=-1$, namely the clustering point, shift towards lower $\varepsilon$ values (redshift) while resonances above $\varepsilon=-1$ shift to larger $\varepsilon$ values (blueshift). The $\varepsilon=-1$ point is here denoted as the clustering point, the point where an infinite number of higher-order multipoles (theoretically) accumulate for the case of a sphere $[6,16,40]$. This shift is related to the sharpness of the cube, since smoother superquadrics deliver a spectrum closer to a sphere, while sharper superquadrics approach the spectrum of a perfect cube.

A perfect cube $(p \rightarrow \infty)$, however, is a rather unrealistic case. First, even for finely constructed (grown) cubes, there is always a finite edge-vertex curvature due to molecular and atomic considerations. From a numerical point of view, an extremely sharp corner introduces certain modeling difficulties, and hence special remedies should be considered (see for example the discussions in $[24,41]$ and the references therein).

The polarizability spectra of the $p=50$ cube are different for the two considered meshes (Figure 3), since the refined mesh gives a shifted version of the spectrum versus the regular one. As a reminder both meshes contain the same number of elements (17) per edge. One possible interpretation of this result is that the edge-vertex refined mesh contains more elements at the vicinity of the edges and vertices, thus enhancing the numerical accuracy over regions where strong variations on the surface potential distributions occur. On the other hand, extremely sharp corners consisting of negative permittivity pose severe numerical difficulties regarding their numerical modeling [42].

A comparison between the surface potential distribution on the regular and refined meshes reveals another, mesh-related, discrepancy. The fields of the $C_{4}$ resonance are somewhat smoother for the refined mesh compared with the regular mesh. This is a clear indication that the mesh not only affects the position of the resonances but also the resulting distribution of the surface potential. Actually, the resonances between $C_{2}$ and $C_{5}$, i.e., higher-order resonances, are more sensitive to the mesh setup. This is a direct evidence that higher-order modes pose computational difficulties, even for well-established numerical methodologies.

\section{Historical Remarks: Plasmonic Resonances of a Cube (Years 1970-2004)}

The first theoretical study of the resonant spectrum of a plasmonic cube was perhaps given in 1971 by van Gelder et al. in [10], where the authors recognized the absence of a rigorous theoretical analysis on the subject. The main contribution of their article is the formulation of an eigenproblem in a variational analysis manner, corresponding to a normal-mode analysis for the spectrum of the cube. The estimated resonances are organized in Table 2. A similar, but more complete analysis of the normal modes of a cube was carried out a few years later (1976 and 1977) by Langbein in [29,40]; the results of this study appear also in Table 2. Both works have received little attention up to date.

Undoubtedly, a seminal contribution for the theoretical evaluation of a cube was given in 1975 by Fuchs [11]. This work is the second to appear (after van Gelder), but the first to introduce interesting observations and sketches of the surface distribution of the most pronounced normal modes. Indeed, Fuchs observed and argued that the main six resonances are responsible for $96 \%$ of the observed plasmonic spectrum, deriving a corresponding sum rule to support his argument. Fuchs also draws the connection between even and odd modes. Since then, this work is a reference text that is cited 
by the majority of the contributions related to the resonant spectrum of a cube. The main resonances predicted by Fuchs are also given in Table 2. Finally, the 1970s included a few interesting contributions for the wedge problem [43-46], and the polarization of a dielectric cube by Herrick and Senior [47], which, however, did not touch the plasmonic resonant perspectives.

Table 2. Comparative table of the position of the plasmonic resonances of a cube of permittivity $\varepsilon$.

\begin{tabular}{cccccc}
\hline \multirow{2}{*}{ Modes } & $\begin{array}{c}\text { Van Gelder } \\
\text { et al. (1972) [10] }\end{array}$ & $\begin{array}{l}\text { Fuchs } \\
\mathbf{( 1 9 7 5 )}[11]\end{array}$ & $\begin{array}{c}\text { Langbein } \\
\mathbf{( 1 9 7 6 )}[29]\end{array}$ & $\begin{array}{c}\text { Ruppin } \\
\mathbf{( 1 9 9 6 )} \text { [30] }\end{array}$ & $\begin{array}{c}\text { Sihvola } \\
\text { et al. (2004) [12] }\end{array}$ \\
\hline$C_{1}$ & - & -3.68 & -3.831 & -4.1813 & -4.369 \\
$C_{2}$ & -2.443 & -2.37 & -2.35 & -2.982 & -2.432 \\
$C_{3}$ & -1.323 & -1.9 & -1.941 & -2.401 & - \\
$C_{4}$ & -1.105 & -1.27 & -1.793 & -1.525 & - \\
$C_{5}$ & -1.077 & - & -1.481 & - & - \\
$C_{6}$ & -1.03 & - & -1.304 & - & - \\
$C_{7}$ & -1.017 & - & -1.114 & - & - \\
$C_{8}$ & -1.001 & - & - & - & - \\
\hline cluster point & & & & & -0.808 \\
\hline$C_{9}$ & -0.963 & - & -0.912 & - & -0.424 \\
$C_{10}$ & -0.793 & -0.78 & -0.792 & -0.653 & -0.391 \\
$C_{11}$ & - & -0.42 & -0.57 & - & \\
\hline
\end{tabular}

While the 1980s were a rather silent period, in 1996 Ruppin repeated the calculations for a cube with improved accuracy, verifying qualitatively the results of Fuchs [30]. This work made obvious that the old techniques should be revisited and implemented in new computational platforms. At the same time, the book by Kreibig and Vollmer [48] was published, which is a seminal book for the developments in the optical properties of metal clusters, including the cube.

The 2000s and 2010s witnessed an increased interest on the plasmonic and dielectric properties of the cube, and generally the theoretical modeling of the plasmonic resonances. For example, works by Mayergoyz et al. [49] and Hohenester [50] opened the way for the systematic study of the numerical modeling of arbitrary plasmonic particles. The work done by Sihvola et al. [12] delivered several expressions of the polarizability of the Platonic solids, including the cube, for the dielectric domain [12]. Surprisingly, these expressions contained the approximative position for the main four plasmonic resonances [20] (see Table 2).

In parallel, many other interesting contributions appeared: Helsing and Perfekt refined the accuracy of the polarizability for the cube [17] and other interesting features of the cubes have been recently investigated [25-27,51-53]. Finally, the works by Zhang [13], Cortie [14], Hung [15], and Klimov [16] contain the visualization of the six resonances, evaluated with increased accuracy. In our article we use these four works as a reference for the analysis of the main resonant features of the cube.

\section{Categorization of the Resonances}

Before going into any details, we need to establish a comparative scheme that will facilitate the categorization of the surface potential exerted at the plasmonic range of a rounded cube. Fuchs, Hung et al. and Klimov et al. argued that there is a similarity between the modes on a plasmonic cube and the modes of a plasmonic wedge: both exhibit "even" and "odd" resonances. For a wedge, the even modes appear at the interval between $-3<\varepsilon<-1$, while the odd modes at $-1<\varepsilon<-1 / 3$. The cube is a solid with solid vertex angle of $\pi / 2$, and hence it is intuitive to approach the cube as a 3D wedge problem.

Departing from the sphere, whose resonances reside at the $-2 \leq \varepsilon \leq-1$ range, one observes that the superquadric perturbation causes either the redshift or the blueshift of a given resonance, with the actual physical mechanism being unknown. Since the amount of multipole resonances of a sphere 
is theoretically infinite, a cube also exerts infinite amount of resonances. These effects lead to the conclusion that the resonances can be associated with an even (redshift) and an odd (blueshift) parity.

Both smooth and sharp cases revealed resonances with surface distribution along their edge and face. At this point a reasonable question emerges: can we categorize all the resonances using any symmetry or other considerations? Hung et al. [15] proposed a simple characterization scheme that follows the number of the nodes, i.e., points between negative and positive surface potential distribution, at the three edges of cube, $\left\{N_{x}, N_{y}, N_{z}\right\}$. For example, the first resonance of the $p=50$ case $C_{1}$ reads $\{0,0,1\}$, second $C_{2}\{2,2,3\}$ and third $C_{3}\{4,4,5\}$. However, this categorization suffers from two drawbacks. The first is that due to symmetry considerations of the excitation field, all resonances have azimuthal rotational symmetry. Hence $N_{x}=N_{y}$ for all resonances and therefore the use of three nodal numbers is redundant. Second, this categorization cannot include resonances with edge-face $\left(C_{4}\right)$ or face $\left(C_{6}\right)$ distributions, since it only covers the nodes at the edges. Apart from [15], there is no other work available in the literature that categorizes the resonances of a cube.

Here, we propose a new categorization scheme based on both mathematical and empirical considerations. First, a cube is a solid that belongs to the octahedral $O_{h}$ symmetry group: there are in total 48 symmetries, 24 due to rotations and 24 due to reflection/translation over certain axes. The available symmetries are further reduced due to the symmetries of the excitation. In our case, the excitation consists of a constant electrostatic field in the $\mathbf{u}_{z}$ direction, exhibiting azimuthal symmetry. Hence we expect that the azimuthal surface potential variations should be azimuthally-symmetric. Moreover, we expect that the reflection symmetries of all planes perpendicular to the $x-y$ plane should be preserved. In addition, we need to account for the variations on both edges and faces.

Figure 4 depicts the proposed categorization scheme. We introduce four different vectors, $\left\{\mathbf{V}_{1}, \mathbf{V}_{2}, \mathbf{F}_{1}, \mathbf{F}_{2}\right\}$. The amount of nodes for each vector direction gives a specific number. For example the first six resonances for the $p=15$ case are: $C_{1}:\{1,0,1,0\}, C_{2}:\{3,2,1,0\}, C_{3}:\{3,4,1,0\}, C_{4}$ : $\{1,0,3,2\}, C_{5}:\{0,0,3,2\}$, and $C_{6}:\{0,0,1,0\}$. Similarly, the $p=50$ case gives: $C_{1}:\{1,0,1,0\}, C_{2}$ : $\{3,2,1,0\}, C_{3}:\{5,4,1,0\}, C_{4}:\{3,2,3,2\}, C_{5}:\{0,0,3,2\}$ and $C_{6}:\{0,0,1,0\}$.

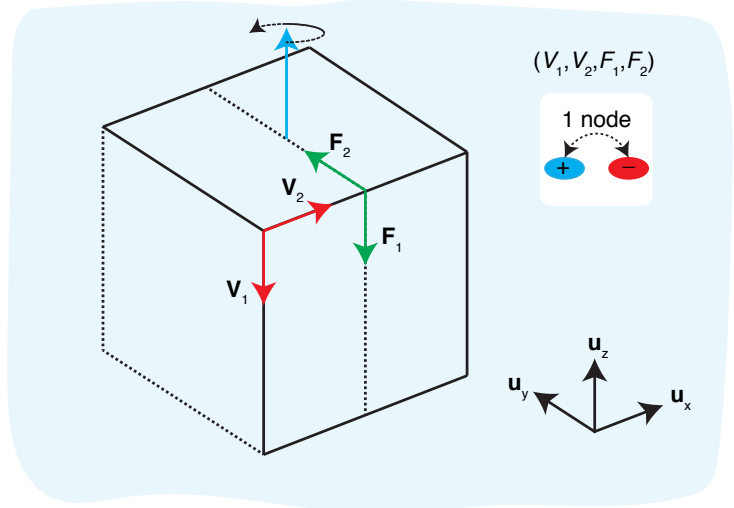

Figure 4. A sketch describing the proposed categorization scheme: vertex-edge vectors $\mathbf{V}_{1}$ and $\mathbf{V}_{2}$ are denoted with red, and face (hedral) vectors $\mathbf{F}_{1}$ and $\mathbf{F}_{2}$ with green. The blue vector indicates the axis of rotational symmetry due to the used excitation ( $\mathbf{u}_{z}$-polarized electrostatic field). A mode is characterized by four numbers, denoting the number of nodes (sign changes of the surface potential distribution) along the four vectors $\left(V_{1}, V_{2}, F_{1}, F_{2}\right)$. The combination of the four numbers and the particular symmetries of cube, allow the recreation of every mode. Please note that in the case of a sphere all $\mathbf{V}_{1}, \mathbf{F}_{1}$, and $\mathbf{F}_{2}$ are parallel (or antiparallel) representing the elevation vector $\mathbf{u}_{\theta}$, while $\mathbf{V}_{2}$ becomes the azimuthal vector $\mathbf{u}_{\phi}$.

Due to symmetry considerations, $V_{1}$ and $F_{1}$ contain always an odd number, while $V_{2}$ and $F_{2}$ contain an even number of nodes. Additionally, categorization number of the resonances below the clustering point $\varepsilon=-1$ sum up to an even number since $V_{1}+V_{2}+F_{1}+F_{2}$ is an even number. In a 
similar manner the categorization number of the resonances above the clustering point sum up to an odd number. This resembles the even and odd parity of the modes found in the 2D plasmonic wedge problem, where the sharpening of the angle of the wedge results in a redshift of the even resonances while odd resonances blueshift. In our considered case, even resonances redshift while odd resonances blueshift $[16,42,44,45]$. We argue that for both cases, the resonances below the clustering point $(\varepsilon<-1)$ give an even categorization number, while resonances above the cluster point $(\varepsilon>-1)$ are characterized as odd.

One interesting feature of this observation is the following: departing from a sphere $p=1$, where all resonances occur below the clustering point $(\varepsilon<-1)$, a rounded cube exhibits resonances that redshift and blueshift, i.e., spread above and below the clustering point $[6,54]$. A similar phenomenon occurs for the spherical core-shell structures where the main symmetric dipole resonance redshifts and the secondary antisymmetric resonance blueshifts as the shell thickness decreases. We speculate that the even and odd resonances on a rounded cube share the same symmetric-antisymmetric property, i.e., even resonances redshift while odd resonances blueshift as the sharpness of the cube is increased.

\section{Discussion and Conclusions}

Equipped with the introduced categorization tool, we present an in-depth investigation of the nature of the surface potential distribution for both smooth and sharp cases. First, we define three main resonant regions, denoted as Cluster 1, 2, and 3, as shown in Figures 5 and 6. Each cluster contains resonances that exhibit a certain character.

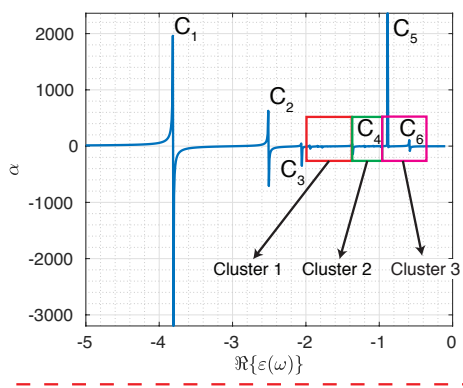

Cluster 3: (no $\mathbf{V}_{1}$ and $\mathbf{V}_{2}$ variation)

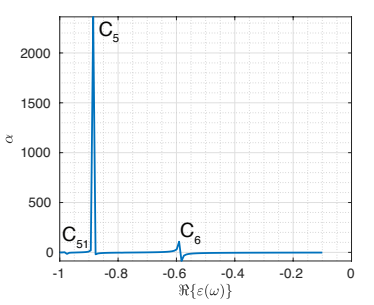

$\mathrm{C}_{51}=(0,0,5,4)$

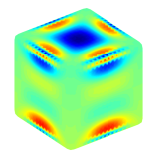

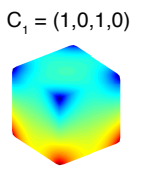
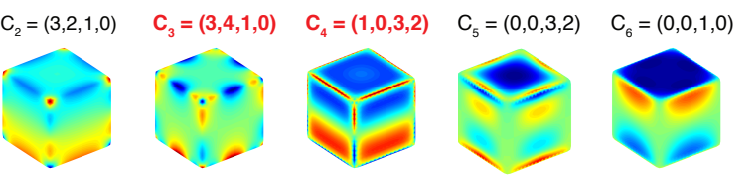

Cluster 1: (no $F_{2}$ variation)
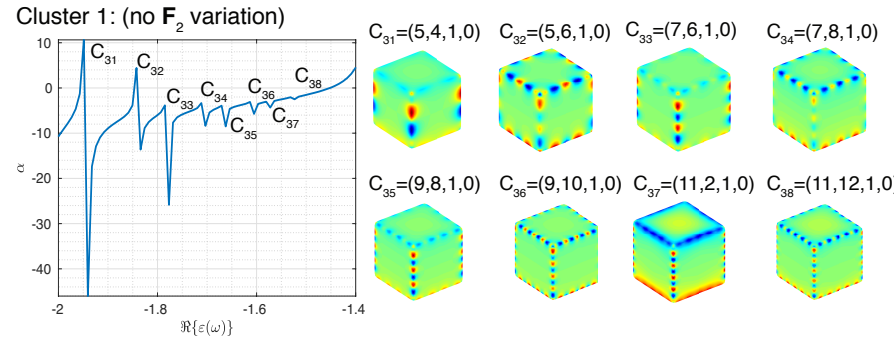

$C_{36}=(9,10,1,0) \quad C_{37}=(11,2,1,0) \quad C_{38}=(11,12,1,0)$
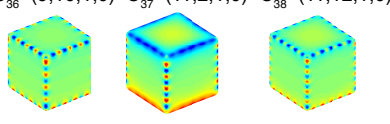

Cluster 2: all possible variations (+spurious)
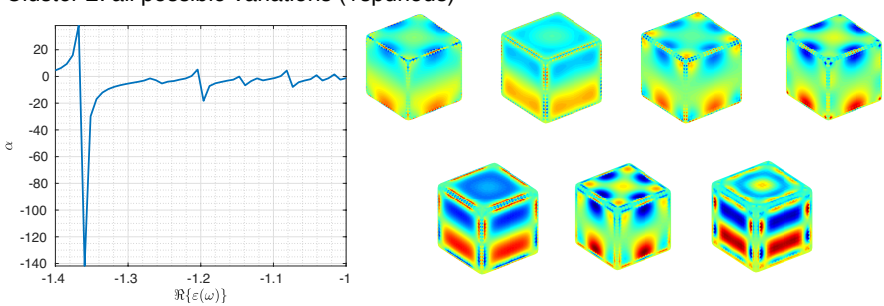

Figure 5. The six main resonances and the three particular clusters for the case of a smooth cube with $p=15$ and a regular mesh. A total of 22 resonances and their surface potential distribution is presented. Above each resonance the categorization number is given, described in Figure 4 . Please note that all resonances in clusters are more sensitive to modeling mesh density, than the main six resonances $\mathrm{C}_{1}-\mathrm{C}_{6}$.

In particular, Cluster 1 contains resonances with nodes across the edges. These resonances are of the form $\left\{V_{1}, V_{2}, 1,0\right\}$, since due to symmetry reasons there is one node at the $F_{1}$ direction and $F_{2}$ is always zero. An interesting point is that all $C_{1}, C_{2}$, and $C_{3}$ belong to this category. We observe that 
the third resonance $C_{3}$ can be different with respect to the sharpness of the cube. For the smooth case we have $C_{3}=\{3,4,1,0\}$ and the next resonance is $C_{31}=\{5,4,1,0\}$ (see Figure 5), while for the sharp case we have $C_{3}=\{5,4,1,0\}$ with the resonance $\{3,4,1,0\}$ being absent. This finding suggests that the development of the nodes at the edges of a cube is very much affected by its sharpness. It is also evident that higher-order modes belonging to Cluster 1 are extremely sensitive to the mesh density and sweeping parameter finesse. In the case of realistic materials most of these higher-order modes practically disappear from the spectrum, since the losses dampen significantly their amplitude.

The aforementioned finding can explain an observed discrepancy between the works of Zhang et al. and Cortie et al. [13,14], and Hung et al. and Klimov et al. [15,16]. In the first case both works report a $C_{3}$ resonance with $\{3,4,1,0\}$ distribution, while the second case the $C_{3}$ resonance is $\{5,4,1,0\}$. This apparent disagreement can be simply explained by the used sharpness, since $[13,14]$ used smoother cubes than the ones used in $[15,16]$.
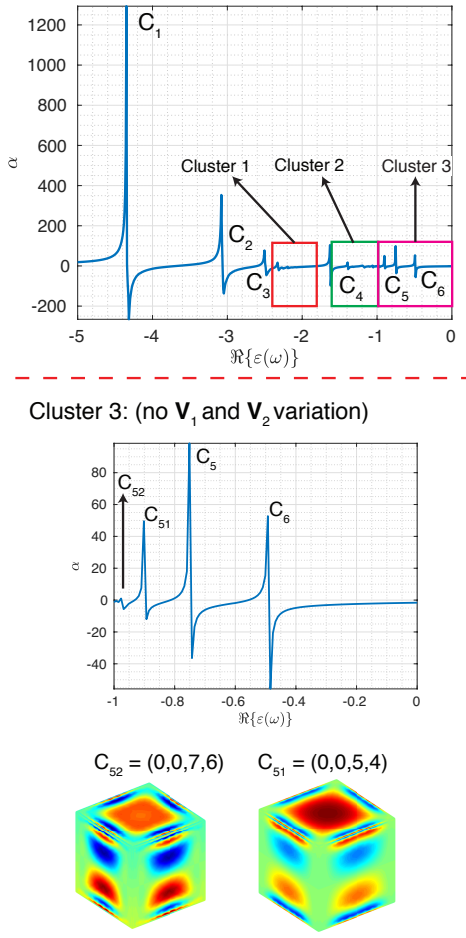
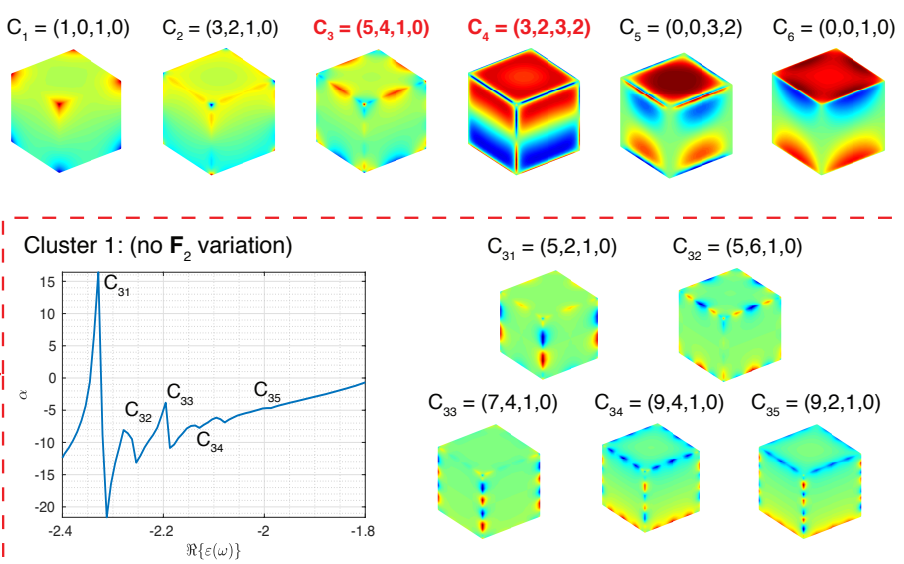

Cluster 2: all possible variations (+spurious)

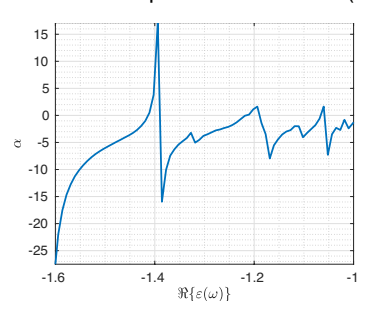

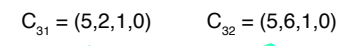

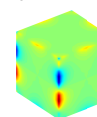

$C_{33}=(7,4,1,0) \quad C_{34}=(9,4,1,0) \quad C_{35}=(9,2,1,0)$
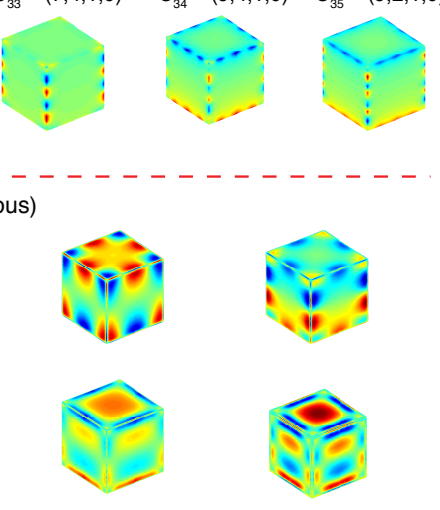

Figure 6. The six main resonances and the three particular clusters for the case of a sharp cube with $p=50$ and a refined mesh. A total of 17 resonances and their surface potential distributions are presented. Above each resonance the categorization number is given, described in Figure 4. Please note that all resonances in clusters are more sensitive to modeling mesh density, than the main six resonances $\mathrm{C}_{1}-\mathrm{C}_{6}$.

Cluster 2 consists of resonances with essentially all possible variation in all four node vectors, as can be seen in Figures 5 and 6. This region is also extremely sensitive to the meshing, revealing a few "pathological" surface distributions, which we characterize as spurious. The main resonance that belongs to this region with a somewhat significant contribution is the $C_{4}$ resonance. Its surface distribution, and therefore its characterization, depends on the mesh density as can be seen in Figures 5 and 6 . For instance, a smoother cube with regular mesh gives $C_{4}=\{1,0,3,2\}$, while the sharper case with a refined mesh exhibits a $\{3,2,3,2\}$ distribution.

Cluster 3 spans above the clustering point $\varepsilon=-1$. The resonances here exhibit distributions with variations only in the $F_{1}$ and $F_{2}$ directions, hence exhibiting an odd parity. Both smooth and sharp cases agree on the surface potential distributions of the most pronounced ones. These resonances can be seen as the equivalent of the antisymmetric resonances of a core-shell structure. This feature 
can be associated with a "self-hybridization" model for a solid particle, analogous to the core-shell hybridization concept [6,55].

Finally, we present a comparison between the four reference works, i.e., Zhang et al., Cortie et al., Hung et al., and Klimov et al. [13-16], and our current analysis for both smooth and sharp cases. The results are classified with the categorization scheme introduced earlier and are summarized in Table 3 .

Table 3. Electrostatic resonances of a cube: Recent contributions

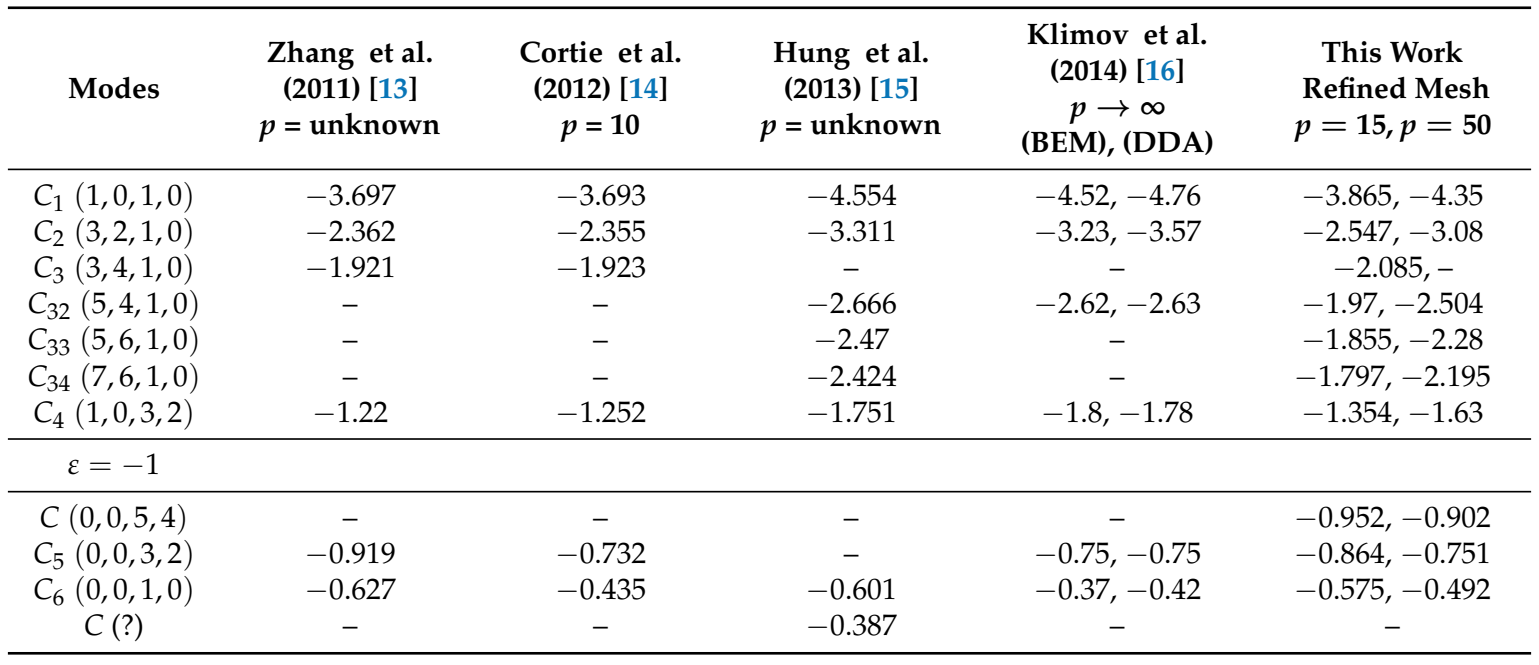

As can be seen from Table 3, both Zhang et al. and Cortie et al. simulated a rather smooth cube, very close to our $p=15$ results. On the other hand, Hung et al. and Klimov et al. simulated a sharp version, and hence their results are close to the $p=50$ case. For the sharp case, the convergence of the leading resonant dipole peak $C_{1}$ (and the higher-order modes) is still an open problem, since the sharp negative-permittivity corner contains a singularity. For the moment it seems that the main dipole resonance converges at around $\varepsilon \approx-4.5$. It is also clear that different rounding factor values affect the appearance of the higher-order modes, especially for the $\{3,4,1,0\}$ and $\{5,4,1,0\}$ cases. In every case, the resonances appearing inside Clusters 1, 2, and 3 pose numerical challenges, since these resonances are sensitive to both rounding factor and meshing. The analysis presented by Hung et al. suggests the appearance of an extra resonance with a surface distribution similar to the $C_{6}=\{0,0,1,0\}$ resonance. However, this resonance, denoted with $C($ ?) in Table 3, has been identified as a separate resonance neither in our analysis nor reported in previous works.

Author Contributions: The idea for studying the plasmonic properties of superquadric particles initiated jointly by D.T. and A.S. D.T. performed the numerical calculations on a superquadric cube based on a code written by Seppo Järvenpää in [12]. D.T. post-processed the results and wrote the first version of the manuscript. All authors contributed to the analysis of the results and edited the final version of the manuscript. D.T. would like to thank prof. Oded Rabin for bringing in our attention the article [15] and the discrepancy appeared between the third resonance of a cube in the works [13-15] and for the interesting discussions during the Metamaterials 2018 conference. All numerical data are available upon request.

Conflicts of Interest: The authors declare no conflict of interest.

\section{Appendix A}

Table A1 presents the resonant positions for all four studied cases, i.e., case $p=15$ and $p=50$, for regular and refined mesh, respectively. 
Table A1. Summary table of the position of the electrostatic resonances of a cube for two different $p$ values and two different meshes.

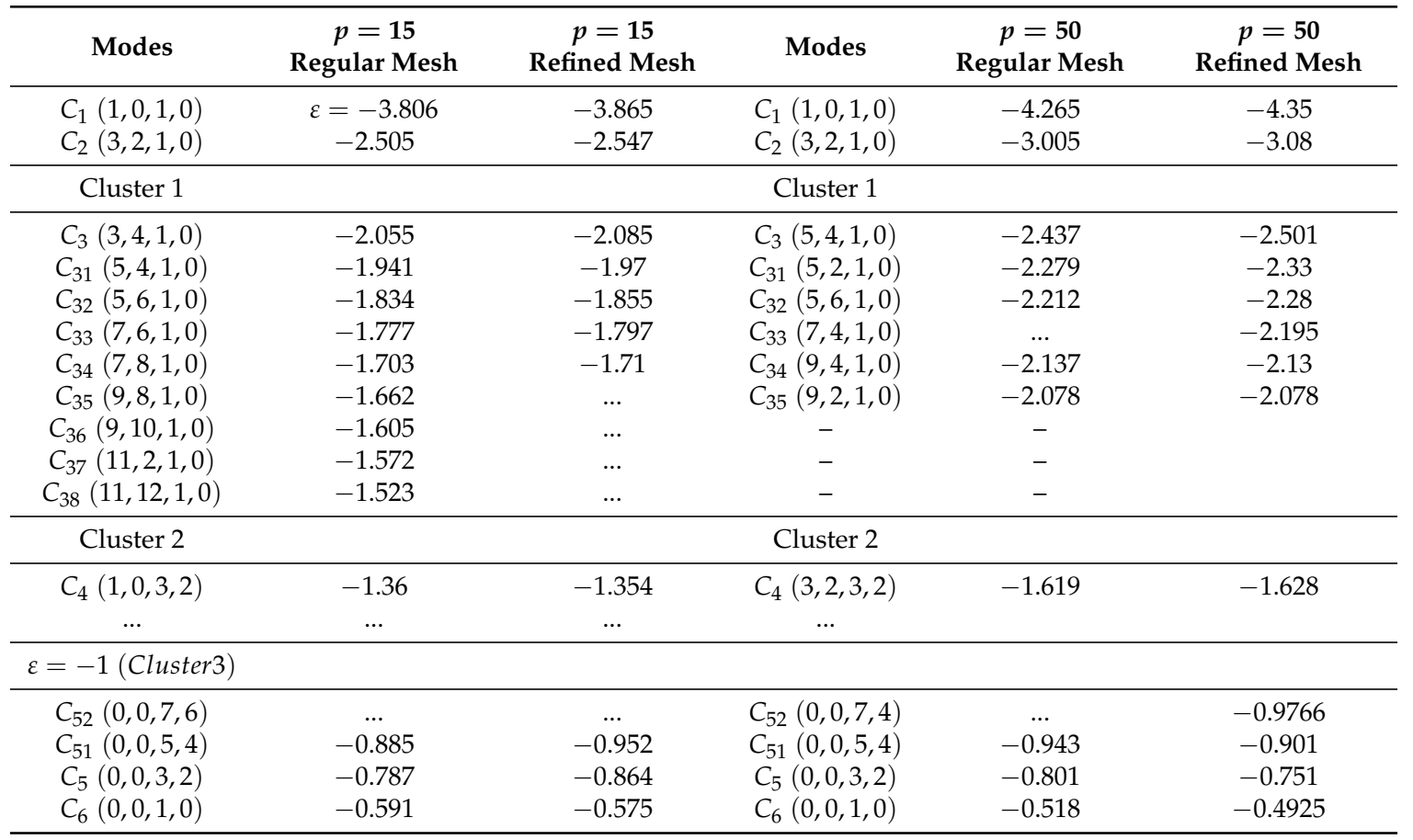

\section{References}

1. Sihvola, A. Electromagnetic Emergence in Metamaterials. In Advances in Electromagnetics of Complex Media and Metamaterials; Zouhdi, S., Sihvola, A., Arsalane, M., Eds.; Springer: Dordrecht, The Netherlands, 2018; pp. 3-17. doi:10.1007/978-94-007-1067-2_1.

2. Staude, I.; Schilling, J. Metamaterial-inspired silicon nanophotonics. Nat. Photonics 2017, 11, 274. [CrossRef]

3. Luk'Yanchuk, B.; Zheludev, N.I.; Maier, S.A.; Halas, N.J.; Nordlander, P.; Giessen, H.; Chong, C.T. The Fano resonance in plasmonic nanostructures and metamaterials. Nat. Mater. 2010. [CrossRef] [PubMed]

4. Akselrod, G.M.; Argyropoulos, C.; Hoang, T.B.; Ciracì, C.; Fang, C.; Huang, J.; Smith, D.R.; Mikkelsen, M.H. Probing the mechanisms of large Purcell enhancement in plasmonic nanoantennas. Nat. Photonics 2014, 8, 835-840. [CrossRef]

5. Kuznetsov, A.I.; Miroshnichenko, A.E.; Brongersma, M.L.; Kivshar, Y.S.; Lukyanchuk, B. Optically resonant dielectric nanostructures. Science 2016, 354, aag2472. [CrossRef] [PubMed]

6. Tzarouchis, D.; Sihvola, A. Light scattering by a dielectric sphere: Perspectives on the Mie resonances. Appl. Sci. 2018, 8, 184. [CrossRef]

7. Tao, A.; Sinsermsuksakul, P.; Yang, P. Polyhedral silver nanocrystals with distinct scattering signatures. Angew. Chem. Int. Ed. 2006, 45, 4597-4601. [CrossRef] [PubMed]

8. Noguez, C. Surface plasmons on metal nanoparticles: The influence of shape and physical environment. J. Phys. Chem. C 2007, 111, 3606-3619. [CrossRef]

9. Zhang, H.; Govorov, A.O. Optical generation of hot plasmonic carriers in metal nanocrystals: The effects of shape and field enhancement. J. Phys. Chem. C 2014, 118, 7606-7614. [CrossRef]

10. Gelder, A.; Holvast, J.; Stoelinga, J.; Wyder, P. Localized polariton modes of small cubic crystals. J. Phys. C Solid State Phys. 1972, 5, 2757. [CrossRef]

11. Fuchs, R. Theory of the optical properties of ionic crystal cubes. Phys. Rev. B 1975, 11, 1732-1740. [CrossRef]

12. Sihvola, A.; Ylä-Oijala, P.; Järvenpää, S.; Avelin, J. Polarizabilities of platonic solids. IEEE Trans. Antennas Propag. 2004, 52, 2226-2233. [CrossRef] 
13. Zhang, S.; Bao, K.; Halas, N.J.; Xu, H.; Nordlander, P. Substrate-induced Fano resonances of a plasmonic nanocube: A route to increased-sensitivity localized surface plasmon resonance sensors revealed. Nano Lett. 2011, 11, 1657-1663. [CrossRef] [PubMed]

14. Cortie, M.B.; Liu, F.; Arnold, M.D.; Niidome, Y. Multimode resonances in silver nanocuboids. Langmuir 2012, 28, 9103-9112. [CrossRef] [PubMed]

15. Hung, L.; Lee, S.Y.; McGovern, O.; Rabin, O.; Mayergoyz, I. Calculation and measurement of radiation corrections for plasmon resonances in nanoparticles. Phys. Rev. B 2013, 88, 75424. [CrossRef]

16. Klimov, V.; Guo, G.Y.; Pikhota, M. Plasmon resonances in metal nanoparticles with sharp edges and vertices: A material independent approach. J. Phys. Chem. C 2014, 118, 13052-13058. [CrossRef]

17. Helsing, J.; Perfekt, K.M. On the polarizability and capacitance of the cube. Appl. Comput. Harmon. Anal. 2013, 34, 445-468. [CrossRef]

18. Raziman, T.V.; Martin, O.J.F. Polarisation charges and scattering behaviour of realistically rounded plasmonic nanostructures. Opt. Express 2013, 21, 21500. [PubMed]

19. Tzarouchis, D.C.; Ylä-Oijala, P.; Ala-Nissila, T.; Sihvola, A. Shape effects on surface plasmons in spherical, cubic, and rod-shaped silver nanoparticles. Appl. Phys. A 2016, 122, 298. [CrossRef]

20. Tzarouchis, D.C.; Ylä-Oijala, P.; Ala-Nissila, T.; Sihvola, A. Plasmonic properties and energy flow in rounded hexahedral and octahedral nanoparticles. J. Opt. Soc. Am. B 2016, 33, 2626. [CrossRef]

21. Ramaccia, D.; Toscano, A.; Bilotti, F. Scattering and absorption from super-spherical nanoparticles: analysis and design for transparent displays [Invited]. J. Opt. Soc. Am. B 2017, 34, D62-D67. [CrossRef]

22. Ramaccia, D.; Arcieri, S.; Toscano, A.; Bilotti, F. Core-shell super-spherical nanoparticles for LSPR-based sensing platforms. IEEE J. Sel. Top. Quantum Electron. 2017, 23, 380-387. [CrossRef]

23. Lagos, M.J.; Trügler, A.; Hohenester, U.; Batson, P.E. Mapping vibrational surface and bulk modes in a single nanocube. Nature 2017, 543, 529. [CrossRef] [PubMed]

24. Tzarouchis, D.C.; Ylä-Oijala, P.; Sihvola, A. Study of plasmonic resonances on Platonic solids. Radio Sci. 2017, 52, 1450-1457. [CrossRef]

25. Cherqui, C.; Li, G.; Busche, J.A.; Quillin, S.C.; Camden, J.P.; Masiello, D.J. Multipolar nanocube plasmon mode-mixing in finite substrates. J. Phys. Chem. Lett. 2018, 9, 504-512. [CrossRef] [PubMed]

26. Ahmad, M. Simulations of the surface plasmon resonances of Au@Ag core-shell nanocubes: Effect of core-shell size ratio. Plasmonics 2018, 13, 981-990. doi:10.1007/s11468-017-0596-z. [CrossRef]

27. Collins, S.M. Dispersion characteristics of face modes in ionic-crystal and plasmonic-metal nanoparticles. Phys. Rev. B 2018, 97, 245422. [CrossRef]

28. Tzarouchis, D.C.; Ylä-Oijala, P.; Sihvola, A. Plasmonic eigenmodes on regular nano-polyhedra: Case study of electrostatic and dynamic resonant effects on rounded superquadric solids. In Proceedings of the 2018 12th International Congress on Artificial Materials for Novel Wave Phenomena (Metamaterials), Espoo, Finland, 27 August 2018-1 September 2018. doi:10.1109/MetaMaterials.2018.8534103.

29. Langbein, D. Normal electric modes at small polyhedral particles. J. Phys. A. Math. Gen. 1977, $10,1031$. [CrossRef]

30. Ruppin, R. Plasmon frequencies of cube shaped metal clusters. Zeitschrift für Phys. D Atoms Mol. Clust. 1996, 36, 69-71. [CrossRef]

31. Loria, G. Spezielle Algebraische und Transzendente Ebene Kurven; Theorie und Geschichte; Teubner: Leipzig, Berlin, Germany, 1911.

32. Jaklic, A.; Leonardis, A.; Solina, F. Segmentation and Recovery of Superquadrics; Springer Science \& Business Media: Berlin/Heidelberg, Germany, 2013; Volume 20.

33. Gardner, M. Piet Hein's Superellipse. In Math. Carnival A New Round-Up Tantalizers Puzzles from Sci. Am.; Vintage: New York, NY, USA, 1977; Chapter 18, pp. 240-254.

34. Barr, A.H. Superquadrics and angle-preserving transformations. IEEE Comput. Graph. Appl. 1981, 1, 11-23. doi:10.1109/MCG.1981.1673799. [CrossRef]

35. Onaka, S. Superspheres: Intermediate shapes between spheres and polyhedra. Symmetry 2012, 4, 336-343. [CrossRef]

36. Miyazawa, T.; Aratake, M.; Onaka, S. Superspherical-shape approximation to describe the morphology of small crystalline particles having near-polyhedral shapes with round edges. J. Math. Chem. 2012, 50, 249-260. [CrossRef] 
37. Hanson, A.J. Hyperquadrics: Smoothly deformable shapes with convex polyhedral bounds. Comput. Vis. Graph. Image Process. 1988, 44, 191-210. [CrossRef]

38. Ylä-Oijala, P.; Tzarouchis, D.C.; Raninen, E.; Sihvola, A. Characteristic mode analysis of plasmonic nanoantennas. IEEE Trans. Antennas Propag. 2017, 65, 2165-2172. [CrossRef]

39. Ylä-Oijala, P.; Wallen, H.; Tzarouchis, D.C.; Sihvola, A. Surface integral equation-based characteristic mode formulation for penetrable bodies. IEEE Trans. Antennas Propag. 2018, 66, 3532-3539. [CrossRef]

40. Langbein, D. Normal modes at small cubes and rectangular particles. J. Phys. A. Math. Gen. 1976, 9, 627-644. [CrossRef]

41. Sekulic, I.; Tzarouchis, D.C.; Ylä-Oijala, P.; Ubeda, E.; Rius, J.M. Enhanced discretization of surface integral equations for resonant scattering analysis of sharp-edged plasmonic nanoparticles. 2018, Under Review.

42. Wallén, H.; Kettunen, H.; Sihvola, A. Surface modes of negative-parameter interfaces and the importance of rounding sharp corners. Metamaterials 2008, 2, 113-121. [CrossRef]

43. Meixner, J. The behavior of electromagnetic fields at edges. IEEE Trans. Antennas Propag. 1972, 20, $442-446$. [CrossRef]

44. Dobrzynski, L.; Maradudin, A.A. Electrostatic edge modes in a dielectric wedge. Phys Rev B 1972, 6, 3810-3815. [CrossRef]

45. Davis, L.C. Electostatic edge modes of a dielectric wedge. Phys. Rev. B 1976, 14, 5523-5525. [CrossRef]

46. Moussiaux, A.; Ronveaux, A.; Lucas, A. Surface plasmon oscillations for different geometrical shapes. Can. J. Phys. 1977, 55, 1423-1433. doi:10.1139/p77-182. [CrossRef]

47. Herrick, D.; Senior, T. The dipole moments of a dielectric cube. IEEE Trans. Antennas Propag. 1977, 25, 590-592. [CrossRef]

48. Kreibig, U.; Vollmer, M. Optical Properties of Metal Clusters; Springer: Berlin/Heidelberg, Germany, 1995; p. 535. doi:10.1007/978-3-662-09109-8.

49. Mayergoyz, I.D.; Fredkin, D.R.; Zhang, Z. Electrostatic (plasmon) resonances in nanoparticles. Phys. Rev. B Condens. Matter Mater. Phys. 2005, 72. [CrossRef]

50. Hohenester, U.; Krenn, J. Surface plasmon resonances of single and coupled metallic nanoparticles: A boundary integral method approach. Phys. Rev. B 2005, 72, 195429. [CrossRef]

51. Sturman, B.; Podivilov, E.; Gorkunov, M. Metal nanoparticles with sharp corners: Universal properties of plasmon resonances. EPL (Europhysics Lett.) 2013, 101, 57009. [CrossRef]

52. Ramaccia, D.; Arcieri, S.; Toscano, A.; Bilotti, F. Super-spherical core-shell nanoparticles: Nanostructured materials enabling applications in the visible regime. In Proceedings of the 2016 10th International Congress on Advanced Electromagnetic Materials in Microwaves and Optics (METAMATERIALS), Chania, Greece, 19-22 September 2016; pp. 298-300. [CrossRef]

53. Yu, R.; Liz-Marzán, L.M.; García de Abajo, F.J. Universal analytical modeling of plasmonic nanoparticles. Chem. Soc. Rev. 2017, 46, 6710-6724. [PubMed]

54. Tzarouchis, D.C.; Sihvola, A. General scattering characteristics of resonant core-shell spheres. IEEE Trans. Antennas Propag. 2018, 66, 323-330. [CrossRef]

55. Prodan, E.; Radloff, C.; Halas, N.J.; Nordlander, P. A hybridization model for the plasmon response of complex nanostructures. Science 2003, 302, 419-422. [CrossRef] [PubMed]

(C) 2019 by the authors. Licensee MDPI, Basel, Switzerland. This article is an open access article distributed under the terms and conditions of the Creative Commons Attribution (CC BY) license (http:/ / creativecommons.org/licenses/by/4.0/). 